\author{
DOROTA BRÓD
}

\title{
On a two-parameter generalization of Jacobsthal numbers and its graph interpretation
}

\begin{abstract}
In this paper we introduce a two-parameter generalization of the classical Jacobsthal numbers $((s, p)$-Jacobsthal numbers). We present some properties of the presented sequence, among others Binet's formula, Cassini's identity, the generating function. Moreover, we give a graph interpretation of $(s, p)$-Jacobsthal numbers, related to independence in graphs.
\end{abstract}

1. Introduction. The Jacobsthal sequence $\left\{J_{n}\right\}$ is defined by the second order linear recurrence

$$
J_{n}=J_{n-1}+2 J_{n-2} \quad \text { for } n \geq 2
$$

with $J_{0}=0, J_{1}=1$. The Binet's formula of this sequence has the following form

$$
J_{n}=\frac{1}{3}\left(2^{n}-(-1)^{n}\right) \quad \text { for } n \geq 0
$$

Moreover, the explicit closed form expression for numbers $J_{n}$ is

$$
J_{n}=\sum_{r=0}^{\left[\frac{n-1}{2}\right]}\left(\begin{array}{c}
n-1-r \\
r
\end{array}\right) 2^{r} \quad \text { for } n \geq 0 .
$$

2010 Mathematics Subject Classification. 11B37, 11C20, 15B36, 05 C69.

Key words and phrases. Jacobsthal numbers, generalized Jacobsthal numbers, Binet's formula, generating function, graph interpretation, Merrifield-Simmons index. 
Other interesting properties of Jacobsthal numbers are given in [6]. There are many generalizations of this sequence in the literature. The second order recurrence (1) has been generalized in two ways: first, by preserving the initial conditions and second, by preserving the recurrence relation. We recall some of such generalizations:

1) $k$-Jacobsthal sequence $\left\{j_{k, n}\right\}[5], j_{k, n+1}=k j_{k, n}+2 j_{k, n-1}$ for $k \geq 1$ and $n \geq 1$ with $j_{k, 0}=0, j_{k, 1}=1$,

2) $k$-Jacobsthal sequence $\left\{J_{k, n}\right\}[3], J_{k, n+1}=J_{k, n}+k J_{k, n-1}$ for $k \geq 1$ and $n \geq 1$ with $J_{k, 0}=0, J_{k, 1}=1$,

3) generalized Jacobsthal $p$-sequence $\left\{J_{p}\right\}[1]$, for any $p \in \mathbb{Z}^{+}$and $n>p+1$ $J_{p}(n)=J_{p}(n-1)+2 J_{p}(n-p-1)$ with initial conditions $J_{p}(1)=$ $J_{p}(2)=\ldots=J_{p}(p+1)=1$,

4) $(s, t)$-Jacobsthal sequence $\left\{\hat{j}_{n}(s, t)\right\}[8], \hat{j}_{n}(s, t)=s \hat{j}_{n-1}(s, t)+2 t \hat{j}_{n-2}(s, t)$ for $n \geq 2$ with $\hat{j}_{0}(s, t)=0$ and $\hat{j}_{1}(s, t)=1$, for real numbers $s, t$, $s>0, t \neq 0$ and $s^{2}+8 t>0$,

5) Jacobsthal sequence $\{J(d, t, n)\}[7], J(d, t, n)=J(d, t, n-1)+t J(d, t$, $n-d)$ for $n \geq d$ with $J(d, t, 0)=1, J(d, t, n)=1$ for $n=1, \ldots, d$, $t \geq 1, d \geq 2$.

In this paper we introduce a new generalization of the classical Jacobsthal numbers. Unlike other variations, this generalization depends on two integer parameters used in the recurrence relation (1). Let $n, s, p \geq 0$ be integers. We define $(s, p)$-Jacobsthal sequence $\left\{J_{n}(s, p)\right\}$ by the following recurrence

$$
J_{n}(s, p)=2^{s+p} J_{n-1}(s, p)+\left(2^{2 s+p}+2^{s+2 p}\right) J_{n-2}(s, p) \text { for } n \geq 2
$$

with initial conditions $J_{0}(s, p)=1, J_{1}(s, p)=2^{s}+2^{p}+2^{s+p}$.

For $s=p=0$ we obtain $J_{n}(0,0)=J_{n+2}$.

We will describe the terms of the sequence $\left\{J_{n}(s, p)\right\}$ explicitly by using a generalization of Binet's formula. Moreover, we will present some identities for $(s, p)$-Jacobsthal numbers, which generalize known results for the classical Jacobsthal numbers.

2. A graph interpretation of $(s, p)$-Jacobsthal numbers. In general we use the standard terminology and notation of graph theory, see [2]. In this section, we will present an interpretation of $(s, p)$-Jacobsthal numbers related to independence in graphs. Let $G$ be a finite, undirected, simple graph with vertex set $V(G)$ and edge set $E(G)$. Recall that a subset $S$ of $V(G)$ is an independent set of $G$ if no two vertices of $S$ are adjacent in $G$. Moreover, every one-element subset of $V(G)$ and the empty set are independent sets of $G$. The number of independent sets of a graph $G$ is denoted by $N I(G)$. In the chemical literature the number of independent sets of a graph $G$ is called the Merrifield-Simmons index of $G$ and is denoted by $\sigma(G)([4])$. The numbers $J_{n}(s, p)$ have the graph interpretation directly related to the Merrifield-Simmons index. 
Consider a graph $H_{n}^{s, p}$ (Figure 1), where $n \geq 1, s, p \geq 0$.

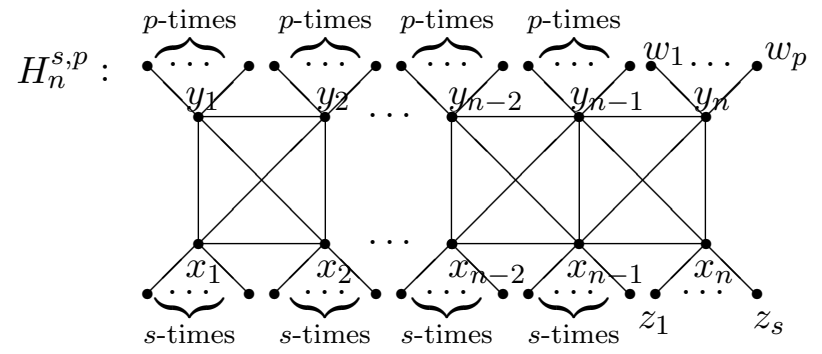

Figure 1.

Theorem 1. Let $n, s, p$ be integers, $n \geq 1, s, p \geq 0$. Then

$$
\sigma\left(H_{n}^{s, p}\right)=J_{n}(s, p) .
$$

Proof. In the beginning we will determine the number of independent sets of graphs $H_{1}^{s, p}$ and $H_{2}^{s, p}$. Assume that vertices of the graphs are numbered as in Figure 1. Denote by $L(x)$ the set of pendant vertices attached to the vertex $x$. Let $n=1$. Assume that $S$ is any independent set of $H_{1}^{s, p}$. Consider two cases.

Case 1. $y_{1} \in S$.

Then $x_{1}, w_{1}, \ldots, w_{p} \notin S$. Hence $S=\left\{y_{1}\right\} \cup Z$, where $Z$ is any subset of the set $\left\{z_{1}, \ldots, z_{s}\right\}$.

Case 2. $y_{1} \notin S$. Consider two possibilities.

\section{1. $x_{1} \in S$.}

Then $S=\left\{x_{1}\right\} \cup W$, where $W$ is any subset of the set $\left\{w_{1}, \ldots, w_{p}\right\}$.

2.2. $x_{1} \notin S$.

Then $S=Z \cup W$.

Finally, we have $\sigma\left(H_{1}^{s, p}\right)=2^{s}+2^{p}+2^{s+p}=J_{1}(s, p)$.

In the same manner we can obtain

$$
\begin{aligned}
\sigma\left(H_{2}^{s, p}\right) & =2^{2 p+s}+2^{p+2 s}+2^{s+p}\left(2^{s}+2^{p}+2^{s+p}\right) \\
& =2^{2 s+p+1}+2^{s+2 p+1}+2^{2 s+2 p}=J_{2}(s, p) .
\end{aligned}
$$

Let $n \geq 3$. Assume that $S$ is any independent set of $H_{n}^{s, p}$. Consider two cases.

Case 1. $y_{n} \in S$.

Let $\mathcal{S}_{1}$ be a family of all independent sets $S$ of the graph $H_{n}^{s, p}$ such that $y_{n} \in S$. Then $x_{n}, x_{n-1}, y_{n-1}, w_{1}, \ldots, w_{p} \notin S$. Hence $S=$ $S^{\prime} \cup\left\{y_{n}\right\} \cup S_{1} \cup S_{2} \cup S_{3}$, where $S^{\prime}$ is any independent set of the graph $H_{n}^{s, p} \backslash\left\{x_{n}, x_{n-1}, y_{n}, y_{n-1}\right\} \backslash\left(L\left(x_{n}\right) \cup L\left(x_{n-1}\right) \cup L\left(y_{n}\right) \cup L\left(y_{n-1}\right)\right)$, isomorphic to $H_{n-2}^{s, p}, S_{1} \subset L\left(x_{n}\right), S_{2} \subset L\left(x_{n-1}\right), S_{3} \subset L\left(y_{n-1}\right)$. Hence by the fundamental combinatorial statements we have $\left|\mathcal{S}_{1}\right|=$ $2^{p} \cdot\left(2^{s}\right)^{2} \sigma\left(H_{n-2}^{s, p}\right)$. 
Case 2. $y_{n} \notin S$.

Let $\mathcal{S}_{2}$ be a family of all independent sets $S$ of the graph $H_{n}^{s, p}$ such that $y_{n} \notin S$. Consider two possibilities.

2.1. $x_{n} \notin S$.

Then $S=S^{\prime \prime} \cup S_{1} \cup S_{4}$, where $S^{\prime \prime}$ is any independent set of the graph $H_{n}^{s, p} \backslash\left\{x_{n}, y_{n}\right\} \backslash\left(L\left(x_{n}\right) \cup L\left(y_{n}\right)\right)$, isomorphic to $H_{n-1}^{s, p}, S_{1} \subset L\left(x_{n}\right)$, $S_{4} \subset L\left(y_{n}\right)$.

2.2. $x_{n} \in S$.

Then $S=S^{\prime} \cup\left\{x_{n}\right\} \cup S_{2} \cup S_{3} \cup S_{4}$, where $S^{\prime}$ is any independent set of the graph $H_{n}^{s, p} \backslash\left\{x_{n}, x_{n-1}, y_{n}, y_{n-1}\right\} \backslash\left(L\left(x_{n}\right) \cup L\left(x_{n-1}\right) \cup L\left(y_{n}\right) \cup\right.$ $\left.L\left(y_{n-1}\right)\right)$, isomorphic to $H_{n-2}^{s, p}$.

Consequently, $\left|\mathcal{S}_{2}\right|=2^{s} \cdot 2^{p} \sigma\left(H_{n-1}^{s, p}\right)+\left(2^{p}\right)^{2} \cdot 2^{s} \sigma\left(H_{n-2}^{s, p}\right)$.

Finally, for $n \geq 3$ we obtain

$$
\sigma\left(H_{n}^{s, p}\right)=\left|\mathcal{S}_{1}\right|+\left|\mathcal{S}_{2}\right|=2^{s+p} \sigma\left(H_{n-1}^{s, p}\right)+\left(2^{2 s+p}+2^{2 p+s}\right) \sigma\left(H_{n-2}^{s, p}\right)
$$

with $\sigma\left(H_{1}^{s, p}\right)=2^{s}+2^{p}+2^{s+p}$ and $\sigma\left(H_{2}^{s, p}\right)=2^{2 s+p+1}+2^{s+2 p+1}+2^{2 s+2 p}$, which ends the proof.

Corollary 2. Let $n \geq 1$. Then $\sigma\left(H_{n}^{0,0}\right)=J_{n}(0,0)=J_{n+2}$.

3. Some identities for $(s, p)$-Jacobsthal numbers. The characteristic equation, associated with the recurrence relation (2) is

$$
r^{2}-2^{s+p} r-\left(2^{2 s+p}+2^{s+2 p}\right)=0
$$

with roots

$$
\begin{aligned}
& r_{1}=2^{s+p-1}+\frac{1}{2} \sqrt{4^{s+p}+2^{s+p+2}\left(2^{s}+2^{p}\right)}, \\
& r_{2}=2^{s+p-1}-\frac{1}{2} \sqrt{4^{s+p}+2^{s+p+2}\left(2^{s}+2^{p}\right)} .
\end{aligned}
$$

Note that

$$
\begin{aligned}
r_{1}+r_{2} & =2^{s+p}, \\
r_{1} r_{2} & =-\left(2^{2 s+p}+2^{s+2 p}\right), \\
r_{1}-r_{2} & =\sqrt{4^{s+p}+2^{s+p+2}\left(2^{s}+2^{p}\right)} .
\end{aligned}
$$

The general formula of $(s, p)$-Jacobsthal sequence can be written by the following identity

$$
J_{n}(s, p)=c_{1} r_{1}^{n}+c_{2} r_{2}^{n}
$$

for some constants $c_{1}, c_{2}$. Using initial conditions $J_{0}(s, p)=1, J_{1}(s, p)=$ $2^{s}+2^{p}+2^{s+p}$, we get the system of two linear equations

$$
\left\{\begin{aligned}
c_{1}+c_{2} & =1 \\
c_{1} r_{1}+c_{2} r_{2} & =2^{s}+2^{p}+2^{s+p} .
\end{aligned}\right.
$$


Solving the system, we obtain

$$
\begin{aligned}
& c_{1}=\frac{2^{s}+2^{p}+2^{s+p}-2^{s+p-1}+\frac{1}{2} \sqrt{4^{s+p}+2^{s+p+2}\left(2^{s}+2^{p}\right)}}{\sqrt{4^{s+p}+2^{s+p+2}\left(2^{s}+2^{p}\right)}}, \\
& c_{2}=\frac{2^{s+p-1}-2^{s}-2^{p}-2^{s+p}+\frac{1}{2} \sqrt{4^{s+p}+2^{s+p+2}\left(2^{s}+2^{p}\right)}}{\sqrt{4^{s+p}+2^{s+p+2}\left(2^{s}+2^{p}\right)}} .
\end{aligned}
$$

Hence we get the following result.

Proposition 3 (Binet's formula). Let $n, s, p \geq 0$. Then the $n$-th $(s, p)$ Jacobsthal number is given by

$$
\begin{aligned}
J_{n}(s, p)= & \frac{\left(2^{s}+2^{p}+2^{s+p}-2^{s+p-1}+\frac{1}{2} \sqrt{\Delta}\right) r_{1}^{n}}{\sqrt{\Delta}} \\
& +\frac{\left(2^{s+p-1}-2^{s}-2^{p}-2^{s+p}+\frac{1}{2} \sqrt{\Delta}\right) r_{2}^{n}}{\sqrt{\Delta}},
\end{aligned}
$$

where $\Delta=4^{s+p}+2^{s+p+2}\left(2^{s}+2^{p}\right), r_{1}=2^{s+p-1}+\frac{1}{2} \sqrt{\Delta}, r_{2}=2^{s+p-1}-\frac{1}{2} \sqrt{\Delta}$.

Using Binet's formula, we can get some identities for $(s, p)$-Jacobsthal numbers.

Theorem 4 (Cassini's identity). Let $n, s, p$ be integers, $n \geq 1, s, p \geq 0$. Then

$$
J_{n+1}(s, p) J_{n-1}(s, p)-J_{n}^{2}(s, p)=(-1)^{n}\left(2^{s}+2^{p}\right)^{2}\left(2^{2 s+p}+2^{s+2 p}\right)^{n-1} .
$$

Proof. By formula (10) we get

$$
\begin{aligned}
J_{n+1}(s, p) & J_{n-1}(s, p)-J_{n}^{2}(s, p) \\
& =\left(c_{1} r_{1}^{n+1}+c_{2} r_{2}^{n+1}\right)\left(c_{1} r_{1}^{n-1}+c_{2} r_{2}^{n-1}\right)-\left(c_{1} r_{1}^{n}+c_{2} r_{2}^{n}\right)^{2} \\
& =c_{1} c_{2} r_{1}^{n+1} r_{2}^{n-1}+c_{1} c_{2} r_{2}^{n+1} r_{1}^{n-1}-2 c_{1} c_{2} r_{1}^{n} r_{2}^{n} \\
& =c_{1} c_{2}\left(r_{1} r_{2}\right)^{n}\left(\frac{r_{1}}{r_{2}}+\frac{r_{2}}{r_{1}}-2\right)=c_{1} c_{2}\left(r_{1} r_{2}\right)^{n-1}\left(r_{1}-r_{2}\right)^{2} .
\end{aligned}
$$

By simple calculations we obtain

$$
c_{1} c_{2}=\frac{-\left(2^{s}+2^{p}\right)^{2}}{4^{s+p}+2^{s+p+2}\left(2^{s}+2^{p}\right)} .
$$

Using formulas (7), (8) and (11), we have

$$
J_{n+1}(s, p) J_{n-1}(s, p)-J_{n}^{2}(s, p)=(-1)^{n}\left(2^{s}+2^{p}\right)^{2}\left(2^{2 s+p}+2^{s+2 p}\right)^{n-1} .
$$

Proposition 5. Let $n, s, p$ be integers, $n \geq 1, s, p \geq 0$. Then

$$
\lim _{n \rightarrow \infty} \frac{J_{n+1}(s, p)}{J_{n}(s, p)}=2^{s+p-1}+\frac{1}{2} \sqrt{4^{s+p}+2^{s+p+2}\left(2^{s}+2^{p}\right)} .
$$


Proof. By formula (10) we have

$$
\lim _{n \rightarrow \infty} \frac{J_{n+1}(s, p)}{J_{n}(s, p)}=\lim _{n \rightarrow \infty} \frac{c_{1} r_{1}^{n+1}+c_{2} r_{2}^{n+1}}{c_{1} r_{1}^{n}+c_{2} r_{2}^{n}}=\lim _{n \rightarrow \infty} \frac{c_{1} r_{1}+c_{2} r_{2}\left(\frac{r_{2}}{r_{1}}\right)^{n}}{c_{1}+c_{2}\left(\frac{r_{2}}{r_{1}}\right)^{n}} .
$$

Since $\lim _{n \rightarrow \infty}\left(\frac{r_{2}}{r_{1}}\right)^{n}=0$, we have

$$
\lim _{n \rightarrow \infty} \frac{J_{n+1}(s, p)}{J_{n}(s, p)}=r_{1}=2^{s+p-1}+\frac{1}{2} \sqrt{4^{s+p}+2^{s+p+2}\left(2^{s}+2^{p}\right)} .
$$

Theorem 6 (summation formula).

$$
\sum_{i=0}^{n-1} J_{i}(s, p)=\frac{J_{n}(s, p)+\left(2^{2 s+p}+2^{s+2 p}\right) J_{n-1}(s, p)-1-2^{s}-2^{p}}{2^{s+p}\left(1+2^{s}+2^{p}\right)-1} .
$$

Proof. By Binet's formula (10) we have

$$
\begin{aligned}
& \sum_{i=0}^{n-1} J_{i}(s, p)=\sum_{i=0}^{n-1}\left(c_{1} r_{1}^{n}+c_{2} r_{2}^{n}\right)=c_{1} \frac{1-r_{1}^{n}}{1-r_{1}}+c_{2} \frac{1-r_{2}^{n}}{1-r_{2}} \\
& =\frac{c_{1}+c_{2}-\left(c_{1} r_{2}+c_{2} r_{1}\right)-\left(c_{1} r_{1}^{n}+c_{2} r_{2}^{n}\right)+r_{1} r_{2}\left(c_{1} r_{1}^{n-1}+c_{2} r_{2}^{n-1}\right)}{\left(1-r_{1}\right)\left(1-r_{2}\right)} \\
& =\frac{c_{1}+c_{2}-\left(c_{1} r_{2}+c_{2} r_{1}\right)-J_{n}(s, p)+r_{1} r_{2} J_{n-1}(s, p)}{1-\left(r_{1}+r_{2}\right)+r_{1} r_{2}} .
\end{aligned}
$$

By formulas (4), (5) and (9) we obtain

$$
c_{1} r_{2}+c_{2} r_{1}=-\left(2^{s}+2^{p}\right) \text {. }
$$

Using (6), (7) and (13), we get

$$
\sum_{i=0}^{n-1} J_{i}(s, p)=\frac{1+2^{s}+2^{p}-J_{n}(s, p)-\left(2^{2 s+p}+2^{s+2 p}\right) J_{n-1}(s, p)}{1-2^{s+p}-2^{2 s+p}-2^{s+2 p}} .
$$

Hence

$$
\sum_{i=0}^{n-1} J_{i}(s, p)=\frac{J_{n}(s, p)+\left(2^{2 s+p}+2^{s+2 p}\right) J_{n-1}(s, p)-1-2^{s}-2^{p}}{2^{s+p}\left(1+2^{s}+2^{p}\right)-1} .
$$

Corollary 7. For $s=p=0$ we get the well-known identity for the classical Jacobsthal numbers

$$
\sum_{i=0}^{n-1} J_{i}=\frac{J_{n+2}+2 J_{n+1}-3}{2} .
$$

The next theorem presents the generating function of $(s, p)$-Jacobsthal sequence. 
Theorem 8. The generating function of the sequence $\left\{J_{n}(s, p)\right\}$ has the following form

$$
f(x)=\frac{1+\left(2^{s}+2^{p}\right) x}{1-2^{s+p} x-\left(2^{2 s+p}+2^{s+2 p}\right) x^{2}} .
$$

Proof. Let $f(x)=\sum_{n=0}^{\infty} J_{n}(s, p) x^{n}$. Then, by recurrence relation (2), we have

$$
\begin{aligned}
f(x)=J_{0}(s, p)+ & J_{1}(s, p) x+\sum_{n=2}^{\infty} J_{n}(s, p) x^{n} \\
=1+\left(2^{s}+\right. & \left.2^{p}+2^{s+p}\right) x \\
& +\sum_{n=2}^{\infty}\left(2^{s+p} J_{n-1}(s, p)+\left(2^{2 s+p}+2^{s+2 p}\right) J_{n-2}(s, p)\right) x^{n} \\
=1+\left(2^{s}+\right. & \left.2^{p}+2^{s+p}\right) x \\
& +2^{s+p} \sum_{n=2}^{\infty} J_{n-1}(s, p) x^{n}+\left(2^{2 s+p}+2^{s+2 p}\right) \sum_{n=2}^{\infty} J_{n-2}(s, p) x^{n} \\
=1+\left(2^{s}+\right. & \left.2^{p}+2^{s+p}\right) x \\
& +2^{s+p} x \sum_{n=1}^{\infty} J_{n}(s, p) x^{n}+\left(2^{2 s+p}+2^{s+2 p}\right) x^{2} \sum_{n=0}^{\infty} J_{n}(s, p) x^{n} \\
=1+\left(2^{s}+\right. & \left.2^{p}+2^{s+p}\right) x \\
& +2^{s+p} x \sum_{n=0}^{\infty} J_{n}(s, p) x^{n}-2^{s+p} x+\left(2^{2 s+p}+2^{s+2 p}\right) x^{2} f(x) .
\end{aligned}
$$

Thus

$$
f(x)=1+\left(2^{s}+2^{p}\right) x+2^{s+p} x f(x)+\left(2^{2 s+p}+2^{s+2 p}\right) x^{2} f(x) .
$$

Hence

$$
f(x)=\frac{1+\left(2^{s}+2^{p}\right) x}{1-2^{s+p} x-\left(2^{2 s+p}+2^{s+2 p}\right) x^{2}},
$$

which ends the proof.

\section{REFERENCES}

[1] Dasdemir, A., The representation, generalized Binet formula and sums of the generalized Jacobsthal p-sequence, Hittite Journal of Science and Engineering 3 (2) (2016), 99-104.

[2] Diestel, R., Graph Theory, Springer-Verlag, Heidelberg-New York, 2005.

[3] Falcon, S., On the $k$-Jacobsthal numbers, American Review of Mathematics and Statistics 2 (1) (2014), 67-77. 
[4] Gutman, I., Wagner, S., Maxima and minima of the Hosoya index and the MerrifieldSimmons index: a survey of results and techniques, Acta Appl. Math. 112 (3) (2010), 323-348.

[5] Jhala, D., Sisodiya, K., Rathore, G. P. S., On some identities for $k$-Jacobsthal numbers, Int. J. Math. Anal. (Ruse) 7 (9-12) (2013), 551-556.

[6] Horadam, A. F., Jacobsthal representation numbers, Fibonacci Quart. 34 (1) (1996), 40-54.

[7] Szynal-Liana, A., Włoch, A., Włoch, I., On generalized Pell numbers generated by Fibonacci and Lucas numbers, Ars Combin. 115 (2014), 411-423.

[8] Uygun, S., The $(s, t)$-Jacobsthal and $(s, t)$-Jacobsthal Lucas sequences, Applied Mathematical Sciences 9 (70) (2015), 3467-3476.

Dorota Bród

Rzeszów University of Technology

Faculty of Mathematics and Applied Physics

al. Powstańców Warszawy 12

35-959 Rzeszów

Poland

e-mail: dorotab@prz.edu.pl

Received March 1, 2018 